Delft University of Technology

\title{
Suppressing Information Diffusion via Link Blocking in Temporal Networks
}

Zhan, Xiuxiu; Hanjalic, Alan; Wang, Huijuan

DOI

10.1007/978-3-030-36687-2_37

Publication date

2020

Document Version

Final published version

Published in

Complex Networks and Their Applications VIII

\section{Citation (APA)}

Zhan, X., Hanjalic, A., \& Wang, H. (2020). Suppressing Information Diffusion via Link Blocking in Temporal Networks. In H. Cherifi, S. Gaito, J. F. Mendes, E. Moro, \& L. M. Rocha (Eds.), Complex Networks and Their Applications VIII : Proceedings of the 8th International Conference on Complex Networks and Their Applications, COMPLEX NETWORKS 2019 (Vol. 1, pp. 448-458). (Studies in Computational Intelligence; Vol. 881 ). Springer. https://doi.org/10.1007/978-3-030-36687-2_37

Important note

To cite this publication, please use the final published version (if applicable).

Please check the document version above.

\footnotetext{
Copyright

Other than for strictly personal use, it is not permitted to download, forward or distribute the text or part of it, without the consent of the author(s) and/or copyright holder(s), unless the work is under an open content license such as Creative Commons.

Takedown policy

Please contact us and provide details if you believe this document breaches copyrights.

We will remove access to the work immediately and investigate your claim.
} 
Green Open Access added to TU Delft Institutional Repository

'You share, we take care!' - Taverne project

https://www.openaccess.nl/en/you-share-we-take-care

Otherwise as indicated in the copyright section: the publisher is the copyright holder of this work and the author uses the Dutch legislation to make this work public. 


\title{
Suppressing Information Diffusion via Link Blocking in Temporal Networks
}

\author{
Xiu-Xiu Zhan, Alan Hanjalic, and Huijuan Wang ${ }^{(凶)}$ \\ Faculty of Electrical Engineering, Mathematics, and Computer Science, \\ Delft University of Technology, Mekelweg 4, 2628 CD Delft, The Netherlands \\ H. Wang@tudelft.nl
}

\begin{abstract}
In this paper, we explore how to effectively suppress the diffusion of (mis)information via blocking/removing the temporal contacts between selected node pairs. Information diffusion can be modelled as, e.g., an SI (Susceptible-Infected) spreading process, on a temporal social network: an infected (information possessing) node spreads the information to a susceptible node whenever a contact happens between the two nodes. Specifically, the link (node pair) blocking intervention is introduced for a given period and for a given number of links, limited by the intervention cost. We address the question: which links should be blocked in order to minimize the average prevalence over time? We propose a class of link properties (centrality metrics) based on the information diffusion backbone [19], which characterizes the contacts that actually appear in diffusion trajectories. Centrality metrics of the integrated static network have also been considered. For each centrality metric, links with the highest values are blocked for the given period. Empirical results on eight temporal network datasets show that the diffusion backbone based centrality methods outperform the other metrics whereas the betweenness of the static network, performs reasonably well especially when the prevalence grows slowly over time.
\end{abstract}

Keywords: Link blocking $\cdot$ Link centrality $\cdot$ Information diffusion backbone $\cdot$ Temporal network $\cdot$ SI spreading

\section{Introduction}

The development of sensor technology and electronic communication service provide us access to rich human interaction data, including proximity data like human face-to-face contacting, electronic communication data like email exchange, message exchange, phone calls $[6,14,18]$. The recorded human interactions can be represented as temporal networks, in which each interaction is represented as a contact at a given time step between two nodes. The availability of such social temporal networks inspires us to explore further how to suppress the diffusion of (mis)information that unfolds on them? One possible intervention is to block the links (i.e., remove contacts between node pairs), but 
only for a given period and given node pairs limited by intervention cost. In this work, we address the question: which links should we block for a given period in order to minimize the prevalence averaged over time, i.e., to prevent or delay the diffusion on temporal networks?

Progress has been made recently in understanding, e.g., nodes with what temporal topological properties (temporal centrality metrics) should be selected as the seed node that starts the information diffusion in order to maximize the final prevalence $[3,5,8,13,15,16]$, links with what temporal topological properties appear more frequently in a diffusion trajectory [19]. These works explored in general the relation between node's or link's topological properties and its role in a dynamic process on a temporal network. Our question which links should be blocked to suppress information diffusion will actually reveal the role of a link within a given period in a diffusion process in relation to the link's temporal topological properties.

As a starting point, we consider the Susceptible-Infected (SI) model as the information diffusion process. A seed node possesses the information (is infected) at time $t=0$ whereas all the other nodes are susceptible. An infected node spreads the information to a susceptible node whenever a contact happens between the two nodes. Given a temporal network within the observation time window $[0, T]$, we would like to choose a given number of links within a period $\left[t_{s}, t_{e}\right]$ to block in order to suppress the diffusion. We propose a comprehensive set of link centrality metrics that characterize diverse temporal topological properties. Each centrality metric is used to rank the links and we remove the links with the highest centrality values for the period $\left[t_{s}, t_{e}\right]$. One group of centrality metrics is based on the information diffusion backbone [19], which characterizes how the contacts appear in a diffusion trajectory thus contribute to the diffusion process. Centrality metrics of the integrated static network, where two nodes are connected if they have at least one contact, are also considered. We propose as well the temporal link gravity, generalized from the static node gravity model [9]. We conduct the SI spreading on the original temporal network as well as the temporal network after link blocking. Their difference in prevalence accumulated over time is used to evaluate the performance of the link blocking strategies/metrics. Our experiments on eight real-world temporal networks show that the diffusion backbone based metrics and the betweenness of the static integrated networks evidently outperform the rest. The backbone based metrics (betweenness of static network) perform(s) better when the prevalence increases fast (slowly) over time. This observation remains universal for diverse choices of the blocking period $\left[t_{s}, t_{e}\right]$ and number of links to block. Our finding points out that both temporal and static centrality metrics, with different computational complexities, are crucial in identifying links' role in a dynamic process.

The rest of the paper is organized as follows. We propose the methodology in Sect. 2. In Sect.2.1, the representation of a temporal network is introduced. In Sect.2.2, the construction of diffusion backbone is illustrated. Afterwards, we propose the link centrality metrics in Sect. 2.3. In Sect. 2.4, the link blocking procedure and the performance evaluation method are given. We further describe 
temporal empirical networks that will be used in Sect. 3. The results of the link blocking strategies on the temporal empirical networks are analyzed in Sect. 4. We conclude our paper in Sect. 5 .

\section{Methods}

\subsection{Representation of Temporal Networks}

A temporal network within a given time window $[0, T]$ is represented as $\mathcal{G}=$ $(\mathcal{N}, \mathcal{L})$, where $\mathcal{N}$ denotes the node set and the number of nodes is $N=|\mathcal{N}|$. The contact set $\mathcal{L}=\{l(j, k, t), t \in[0, T], j, k \in \mathcal{N}\}$ contains the element $l(j, k, t)$ representing that a contact between node $j$ and $k$ occurs at time step $t$. The integrated weighted network of $\mathcal{G}$ is denoted by $G_{W}=\left(\mathcal{N}, \mathcal{L}_{W}\right)$. The weight $w_{j k}$ of link $l(j, k)$ counts the number of contacts between node $j$ and node $k$.

\subsection{Information Diffusion Backbone}

The information diffusion backbone was proposed to characterize how node pairs appear in a diffusion trajectory thus contribute to the actual diffusion process [19]. To illustrate our method, we construct the backbone for the SI model with infection probability $\beta=1$, which means that an infected node infects a susceptible node with probability $\beta=1$ whenever the two nodes have a contact. The backbone can be also constructed for the SI model with any infection probability $\beta \in[0,1]$.

We first record the spreading tree $\mathcal{T}_{i}$ of each node $i$ by setting $i$ as the seed of the SI spreading process starting at $t=0$. The spreading tree $\mathcal{T}_{i}$ is the union of the contacts through which the information propagates. The diffusion backbone $G_{B}$ is defined as the union of all the spreading trees, i.e., $G_{B}=\left(\mathcal{N}, \mathcal{L}_{B}\right)=\bigcup_{i=1}^{N} \mathcal{T}_{i}$. We use $\mathcal{N}, \mathcal{L}_{B}$ to represent the node set and the link set respectively. Each link $l(j, k)$ in $\mathcal{L}_{B}$ is associated with a weight $w_{j k}^{B}$, counting the number of contacts between $j$ and $k$, that appear in diffusion trees/trajectories initiated from every node. An example of how we construct the diffusion backbone $G_{B}$ is given in Fig. 1(a-c).

\subsection{Link Centrality Metrics}

We first propose three backbone based link centrality metrics:

- Backbone Weight. The backbone weight $w_{j k}^{B}$ of a link $l(j, k)$ counts how many times the link or its contacts appear in spreading trees (trajectories) initialized from every node.

- Time-confined Backbone Weight $\left[t_{s}, t_{e}\right]$. Furthermore, we define the timeconfined information diffusion backbone $G_{B^{*}}$, which generalizes our previous backbone definition. The backbone $G_{B^{*}}$ confined within a time window $\left[t_{s}, t_{e}\right]$ 


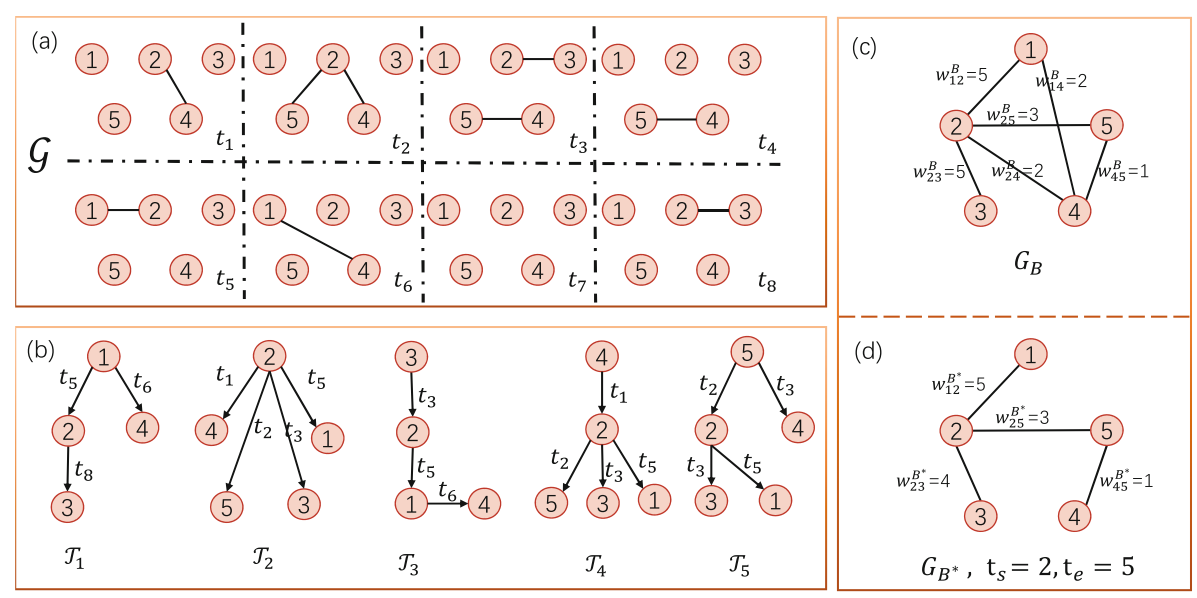

Fig. 1. (a) A temporal network $\mathcal{G}$ with $N=5$ nodes and $T=8$ time steps. (b) Spreading trees rooted at every seed node. The time step on each link denotes the time of the contact through which information diffuses. (c) The diffusion backbone $G_{B}$. (d) Diffusion backbone $G_{B^{*}}$ confined within $t_{s}=2, t_{e}=5$. When we consider the links that only appear in a time window $\left[t_{s}, t_{e}\right]=[2,5]$, the value on the link shows the link weight in $G_{B^{*}}$.

is the union of all the spreading trees but only of the contacts that occur within $\left[t_{s}, t_{e}\right]$. Hence, two nodes in $G_{B^{*}}$ are connected if at least one contact between them within $\left[t_{s}, t_{e}\right]$ appears in a diffusion tree rooted at any node. The weight $w_{j k}^{B^{*}}$ of link $l(j, k)$ in $G_{B^{*}}$ equals to the number of times that contact(s) between $j$ and $k$ within $\left[t_{s}, t_{e}\right]$ that appear in the spreading trees rooted at every node. The link weight in $G_{B^{*}}$ characterizes the frequency that a link, within $\left[t_{s}, t_{e}\right]$, contributes to the information diffusion. An example of the time-confined backbone construction is given in Fig. $1(\mathrm{~d})$, where $t_{s}=$ $2, t_{e}=5$. Take link $l(2,4)$ as an example. It appears in the spreading trees twice, both at time step $t_{1}$, which is beyond range $\left[t_{s}=2, t_{e}=5\right]$. Therefore, $w_{24}^{B^{*}}=0$. Link $l(2,3)$ appears at time step $t_{8}, t_{3}, t_{3}, t_{3}, t_{3}$ in all the spreading trees, only the time step $t_{8}$ is out of range $[2,5]$. Hence, $w_{23}^{B^{*}}=4$.

- Backbone Betweenness. The backbone betweenness is defined to measure the link influence in disseminating global information. Given a spreading tree $\mathcal{T}_{i}$, the number of descendant nodes of link $l(j, k)$ is denoted as $B_{j k}^{i}$. We define the backbone betweenness $B_{j k}$ of link $l(j, k)$ as the average number of descendant nodes over all the spreading trees, i.e., $B_{j k}=\frac{1}{N} \sum_{i \in \mathcal{N}} B_{j k}^{i}$.

We consider as well the following centrality metrics derived from the integrated weighted network. Only the links in the integrated network deserves blocking. All the following metrics are zero for a node pair that they are not connected in the integrated network.

- Degree Product of a link $l(j, k)$ is the product of the degrees of its two end nodes in $G_{W}$, i.e., $d_{j} \cdot d_{k}$. 
- Strength Product. The node strength of a node $j$ in $G_{W}$ is defined as $s_{j}=$ $\sum_{k \in \Gamma_{j}} w_{j k}$, where $\Gamma_{j}$ is the neighbor set of node $j$. Hence, the strength of a node equals to the total weight of all the links incident to this node. We define strength product of a link $l(j, k)$ as $s_{j} \cdot s_{k}$.

- Static Betweenness. The static betweenness centrality for a link is the number of shortest paths between all node pairs that pass through the link. To compute the shortest path, we define the distance of each link in the integrated network $G_{W}$ inversely proportional to its link weight in $G_{W}$. This choice follows the assumption that links with a higher weight in $G_{W}$ can spread information faster [12].

- Link Weight. The link weight $w_{j k}$ of a link $l(j, k)$ in $G_{W}$ tells the total number of contacts between node $j$ and $k$ in the temporal network $\mathcal{G}$ within the observation window $[0, T]$.

- Time-confined Link Weight $\left[t_{s}, t_{e}\right]$ refers to the number of contacts between two ending nodes that occur in $\left[t_{s}, t_{e}\right]$.

- Temporal Link Gravity. The link gravity between node $j$ and $k$ has been defined by regarding the node degree as the mass, the distance $\mathcal{H}_{j k}$ of the shortest path on static network $G_{W}$ between $j$ and $k$ as the distance. The static gravity of node $j$ can be further defined as $\sum_{k \neq j} \frac{d_{j} d_{k}}{\mathcal{H}_{j k}^{2}}$. The static node gravity has been used to select the seed node of an information diffusion process in order to maximize the prevalence [9], motivated by the fact that it contains both the neighborhood and the path information of a node. We generalize the gravity definition to temporal networks. The temporal link gravity of $l(j, k)$ is defined as $\frac{1}{2}\left(\frac{d_{j} d_{k}}{\mathcal{Q}_{j k}^{2}}+\frac{d_{j} d_{k}}{\mathcal{Q}_{k j}^{2}}\right)$, where $\mathcal{Q}_{j k}$ is the number of links of the shortest path from $j$ to $k$ in all the directed spreading trees (see Fig. 1(b)). Specifically, the shortest directed path from $j$ to $k$ is computed in each spreading tree rooted at one seed node. We consider the shortest among these $N$ shortest directed paths and its length (number of links) is $\mathcal{Q}_{j k}$.

\subsection{Link Blocking and Evaluation}

We illustrate the link blocking procedure and the evaluation method to measure the effectiveness of link blocking strategies. Given a temporal network, we specify the time window to block links as $\left[t_{s}, t_{e}\right]$. For each time window $\left[t_{s}, t_{e}\right]$, we count the number of node pairs $\left|\mathcal{L}_{W}^{*}\left(t_{s}, t_{e}\right)\right|$ that have at least one contact within $\left[t_{s}, t_{e}\right]$ and block $5 \%, 10 \%, 20 \%, 40 \%, 60 \%, 80 \%$ and $100 \%$ of $\left|\mathcal{L}_{W}^{*}\left(t_{s}, t_{e}\right)\right|$ links respectively using each centrality metric. The number of links to be blocked is further expressed as the fraction $f$ of the number of links in the integrated network. For each centrality metric, we block the given fraction $f$ of links that have the highest values for the given period $\left[t_{s}, t_{e}\right]$, i.e., remove all the contacts within $\left[t_{s}, t_{e}\right]$ associated with the selected links.

We perform the SI spreading model by setting each node as the seed node on the original temporal network as well as the temporal network after the link blocking. The average prevalence is the average over each possible seed node. The average prevalence of the SI diffusion at any time $t$ when the selected fraction 
$f$ of links are blocked within $\left[t_{s}, t_{e}\right]$ and when no links are blocked is denoted as $\rho_{f}(t)$ and $\rho_{o}(t)$ respectively, where $t \in[0,1, \ldots, T]$. The effectiveness of each centrality metric is evaluated by

$$
\rho_{D}(f)=\frac{\sum_{t=1}^{T}\left(\rho_{0}(t)-\rho_{f}(t)\right)}{\sum_{t=1}^{T} \rho_{0}(t)}
$$

which corresponds to the area below the original prevalence $\rho_{o}(t)$ and above the prevalence curve $\rho_{f}(t)$ with link blocking normalized by the area under $\rho_{o}(t)$ (shown in Fig. 2(b)). A larger $\rho_{D}(f)$ implies a more effective link block strategy in suppressing the SI spreading.

\section{Data Description}

In this paper, we use eight temporal network datasets to investigate the link blocking problem in temporal networks. The dataset can be classified into two categories according to the contact type, i.e., proximity (Haggle [1], HighSchool 2012(HS2012) [4], HighSchool2013(HS2013) [10], Reality Mining (RM) [2], Hypertext 2009(HT2009) [7], Primary School(PS) [17] and Infectious [7]) and electronic communication (Manufacturing Email(ME) [11]). The detailed topological features of these datasets are shown in Table 1, including the number of nodes, time steps, contacts, the number of links, link density, average degree and average link weight in $G_{W}$.

On each temporal network, we perform the SI spreading process starting at every node as the seed. The average prevalence $\rho$ over time for each dataset is shown in Fig. 2(a), where the time step is normalized by the time span $T$ of the observation time window. The spreading speed, i.e., how fast the prevalence grows over time, is quite different across networks. Two networks (Haggle and infectious) show slow and relative linear increase in prevalence over times, due to the low link density in these two networks (Table 1). However, the prevalence in the other networks, increases dramatically at the early stage of the spreading process and converges to about $100 \%$.

\section{Empirical Results}

In this section, we evaluate the effectiveness of using aforementioned centrality metrics to select the links to be blocked within $\left[t_{s}, t_{e}\right]$. We consider diverse time windows $\left[t_{s}, t_{e}\right]$ as listed in Table 2 . Intervention is possibly introduced at different diffusion phases. Hence, $t_{s} \in\left\{T_{10 \% I}, T_{20 \% I}, T_{30 \% I}, T_{40 \% I}, T_{50 \% I}\right\}$, where $T_{10 \% I}$ is the time when the average prevalence without blocking reaches $\rho=10 \%$ (see Fig. 2(a)). The duration of each time window is set as the duration for the average prevalence to increase $10 \%$ just before $t_{s}$. If $t_{s}=T_{20 \% I}$, the duration of the time window is $t_{e}-t_{s}=T_{20 \% I}-T_{10 \% I}$. If $t_{s}=T_{10 \% I}$, the duration of the time window is $t_{e}-t_{s}=T_{10 \% I}-T_{0 \% I}=T_{10 \% I}$. The number of links to block has also been chosen systematically. We take $\left[t_{s}=T_{10 \% I}, t_{e}=2 T_{10 \% I}\right]$ as an 
Table 1. Basic properties of the empirical networks. The number of nodes $(N)$, the original length of the observation time window ( $T$ in number of steps), the total number of contacts $(|\mathcal{L}|)$ and the number of links $\left(\left|\mathcal{L}_{W}\right|\right)$, link density, average node degree $(\langle d\rangle)$ and average link weight $\langle w\rangle$ in $G_{W}$ are shown.

\begin{tabular}{l|r|r|r|r|l|l|r}
\hline Network & \multicolumn{1}{l|}{$N$} & \multicolumn{1}{l|}{$T$} & $|\mathcal{L}|$ & $\left|\mathcal{L}_{W}\right|$ & Link density & $\langle d\rangle$ & $\langle w\rangle$ \\
\hline Haggle & 274 & 15,662 & 28,244 & 2,124 & 0.0568 & 15.50 & 13.30 \\
\hline HS2012 & 180 & 11,273 & 45,047 & 2,220 & 0.1378 & 24.67 & 20.29 \\
\hline HS2013 & 327 & 7,375 & 188,508 & 5,818 & 0.1092 & 35.58 & 32.40 \\
\hline HT2009 & 113 & 5,246 & 20,818 & 2,196 & 0.3470 & 38.87 & 9.48 \\
\hline Infectious & 410 & 1,392 & 17,298 & 2,765 & 0.0330 & 13.49 & 6.26 \\
\hline ME & 167 & 57,791 & 82,876 & 3,250 & 0.2345 & 38.92 & 25.50 \\
\hline PS & 242 & 3,100 & 125,773 & 8,317 & 0.2852 & 68.74 & 15.12 \\
\hline RM & 96 & 33,452 & $1,086,404$ & 2,539 & 0.5568 & 52.90 & 427.89 \\
\hline
\end{tabular}
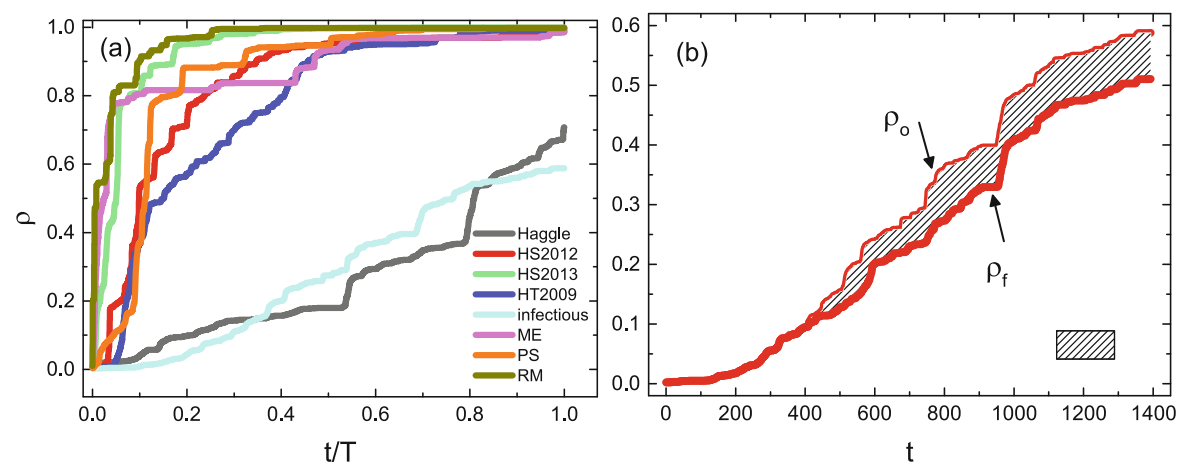

Fig. 2. (a) Evolution of the average prevalence $\rho$ of the SI model $(\beta=1)$ for the eight empirical datasets. (b) An example of the area difference between the original spreading curve $\left(\rho_{o}\right)$ and the curve $\left(\rho_{f}\right)$ after blocking $f$ fraction of links.

example to illustrate our findings. Figure 3 shows the effectiveness of each centrality metric as a function of $f$, which is the number of links blocked normalized by the number of links in the integrated network. The random selection of links from those that have at least one contact within $\left[t_{s}, t_{e}\right]$ is used as a baseline, in which each point is the averaged over 100 realizations.

We find that four link centrality metrics always outperform the random selection: static betweenness, backbone weight, time-confined backbone weight $\left[t_{s}, t_{e}\right]$ and backbone betweenness. In Haggle and infectious, the best performance comes from static betweenness, whereas the time-confined backbone weight $\left[t_{s}, t_{e}\right]$ outperforms the other metrics in the other six networks. Figure 2 shows that the prevalence grows slowly over time in Haggle and infectious. Hence, the static betweenness seems a suitable link blocking strategy for networks with a slow spreading speed. However, for networks where information propagates fast, the 
Table 2. The time window $\left[t_{s}, t_{e}\right]$ we choose for link blocking based on the average prevalence $\rho$ when $\beta=1$. For instance, $T_{10 \% I}$ represents the time when the prevalence reaches $\rho=0.1$.

\begin{tabular}{l|l|l|l}
\hline Network & {$\left[T_{10 \% I}, 2 T_{10 \% I}\right]$} & {$\left[T_{20 \% I}, 2 T_{20 \% I}-T_{10 \% I}\right]$} & {$\left[T_{30 \% I}, 2 T_{30 \% I}-T_{20 \% I}\right]$} \\
\hline Haggle & {$[3293,6586]$} & {$[8416,13539]$} & {$[9523,10630]$} \\
\hline HS2012 & {$[403,806]$} & {$[675,947]$} & {$[925,1175]$} \\
\hline HS2013 & {$[50,100]$} & {$[113,176]$} & {$[195,277]$} \\
\hline HT2009 & {$[332,664]$} & {$[377,422]$} & {$[439,501]$} \\
\hline Infectious & {$[410,820]$} & {$[553,696]$} & {$[751,949]$} \\
\hline ME & {$[168,336]$} & {$[285,402]$} & {$[461,637]$} \\
\hline PS & {$[136,272]$} & {$[276,416]$} & {$[287,298]$} \\
\hline RM & {$[5,10]$} & {$[34,63]$} & {$[111,188]$} \\
\hline Network & {$\left[T_{40 \% I}, 2 T_{40 \% I}-T_{30 \% I}\right]$} & {$\left[T_{50 \% I}, 2 T_{50 \% I}-T_{40 \% I}\right]$} & \\
\hline Haggle & {$[12440,15357]$} & {$[12668,12896]$} & \\
\hline HS2012 & {$[1043,1161]$} & {$[1109,1175]$} & \\
\hline HS2013 & {$[236,277]$} & {$[369,502]$} & \\
\hline HT2009 & {$[568,697]$} & {$[790,1012]$} & \\
\hline Infectious & {$[955,1159]$} & {$[1062,1169]$} & \\
\hline ME & {$[731,1001]$} & {$[1387,2043]$} & \\
\hline PS & {$[323,359]$} & {$[347,371]$} & \\
\hline RM & {$[133,155]$} & {$[257,381]$} & \\
\hline & & & \\
\hline
\end{tabular}

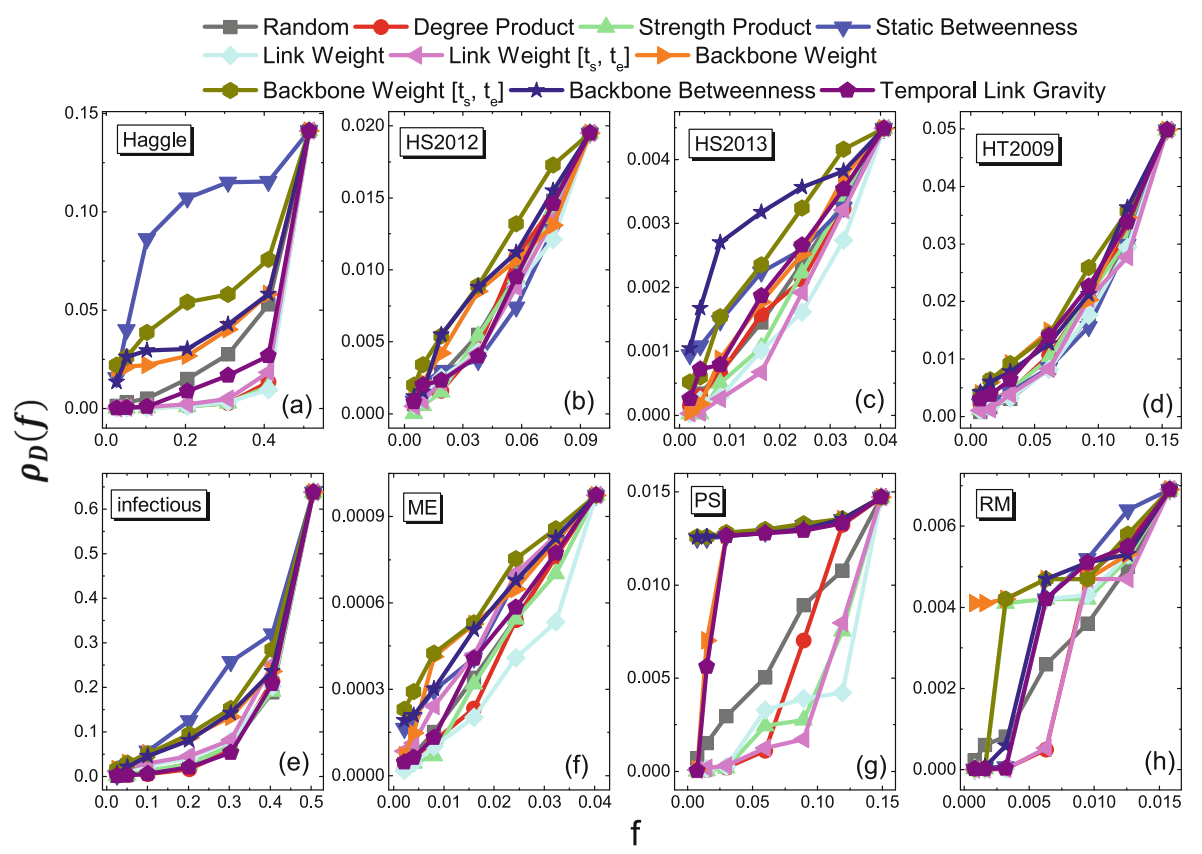

Fig. 3. The effectiveness $\rho_{D}(f)$ of each centrality metric in selecting the links to block within time window $\left[T_{10 \% I}, 2 T_{10 \% I}\right]$. Each point on the curve corresponds to block $5 \%, 10 \%, 20 \%, 40 \%, 60 \%, 80 \%$ and $100 \%$ of $\left|\mathcal{L}_{W}^{*}\left(t_{s}=T_{10 \% I}, 2 T_{10 \% I}\right)\right|$ links, respectively. The $\mathrm{x}$-axis $f$ is obtained by the number of links blocked normalized by the number of links in the integrated network. 


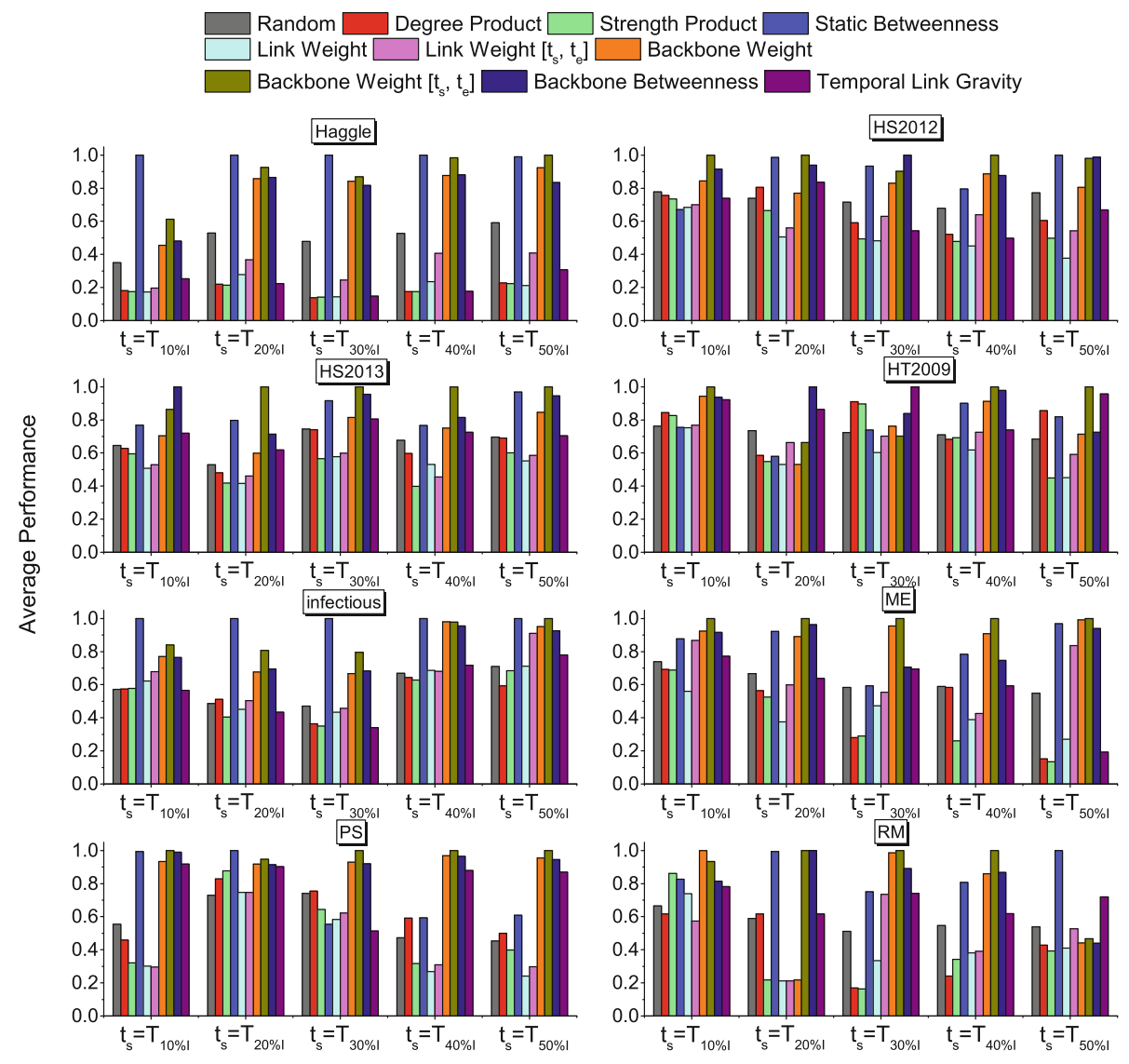

Fig. 4. Average link blocking performance for each centrality metric over different number of blocked links, within different time windows and in different networks. The $\mathrm{x}$ axis shows the time windows. We only show the starting time $t_{s}$ of each time window for simplicity and the ending time of each window can be found in Table 2 .

time-confined backbone weight $\left[t_{s}, t_{e}\right]$ is a good indicator to select the links to block. Furthermore, we find that time-confined link weight $\left[t_{s}, t_{e}\right]$ outperforms link weight and time-confined backbone weight $\left[t_{s}, t_{e}\right]$ outperforms the backbone weight. This implies that considering the link temporal topological features within the blocking time window is crucial for the link selection.

For a given time window $\left[t_{s}, t_{e}\right]$, we define the average performance of a centrality metric as the area under $\rho_{D}(f)$ over the whole range $f$. The average performance is further normalized by the maximal average performance among all the centrality metrics for the given $\left[t_{s}, t_{e}\right]$. This average performance over diverse numbers of links to be blocked allows us to evaluate whether the performance of these centrality metrics is stable when the time window varies. Figure 4 verifies that our findings within $\left[t_{s}=T_{10 \% I}, t_{e}=2 T_{10 \% I}\right]$ from Fig. 3 can be generalized to the other time windows. 


\section{Conclusion}

In this paper, we investigate how different link blocking strategies could suppress the information diffusion process on temporal networks. The spreading process is modeled by the SI model with infection probability $\beta=1$. We propose diverse classes of link centrality metrics to capture different link temporal topological properties, including the information diffusion backbone based metrics and the static link centrality metrics. According to each metric, we select a given number of links that have the highest centrality value and block them for the given period $\left[t_{s}, t_{e}\right]$. The corresponding effect of such link blocking is evaluated via the extent that the prevalence is suppressed over time.

The empirical results from eight temporal network datasets show that four metrics outperform the random link selection, that is, backbone weight, backbone weight $\left[t_{s}, t_{e}\right]$, backbone betweenness and static betweenness. An interesting finding is that the backbone based metrics, especially time-confined backbone weight $\left[t_{s}, t_{e}\right]$, perform well in networks where information gets prevalent fast. However, the static betweenness outperforms in networks where information propagates slowly. These observations hold for different choices of time window and the number of links to be blocked. Our findings point out the importance of both temporal and static centrality metrics in determining links' role in a diffusion process. Moreover, the time-confined metrics that explicitly explore the property/role of the contacts that occur within the time window in the global diffusion process seems promising in identifying the links to block.

In this work, we select links based on the centrality metrics that are derived from the temporal network information over the whole observation window $[0, T]$. Our study unravels actually the relation between links' or contacts' temporal topological properties and their role in a diffusion process. A more challenging question is how to identify the links to block based on the temporal network information observed so far within $\left[0, t_{s}\right]$.

Acknowledgements. This work has been partially supported by the China Scholarship Council (CSC).

\section{References}

1. Chaintreau, A., Hui, P., Crowcroft, J., Diot, C., Gass, R., Scott, J.: Impact of human mobility on opportunistic forwarding algorithms. IEEE Trans. Mob. Comput. 6, 606-620 (2007)

2. Eagle, N., Pentland, A.S.: Reality mining: sensing complex social systems. Pers. Ubiquit. Comput. 10(4), 255-268 (2006)

3. Estrada, E.: Communicability in temporal networks. Phys. Rev. E 88(4), 042811 (2013)

4. Fournet, J., Barrat, A.: Contact patterns among high school students. PLoS One $\mathbf{9}(9)$, e107878 (2014)

5. Grindrod, P., Parsons, M.C., Higham, D.J., Estrada, E.: Communicability across evolving networks. Phys. Rev. E 83(4), 046120 (2011) 
6. Holme, P.: Modern temporal network theory: a colloquium. Eur. Phys. J. B 88(9), $234(2015)$

7. Isella, L., Stehlé, J., Barrat, A., Cattuto, C., Pinton, J.F., Van den Broeck, W.: What's in a crowd? Analysis of face-to-face behavioral networks. J. Theor. Biol. 271(1), 166-180 (2011)

8. Li, C., Li, Q., Van Mieghem, P., Stanley, H.E., Wang, H.: Correlation between centrality metrics and their application to the opinion model. Eur. Phys. J. B $\mathbf{8 8}(3), 65(2015)$

9. Li, Z., Ren, T., Ma, X., Liu, S., Zhang, Y., Zhou, T.: Identifying influential spreaders by gravity model. Sci. Rep. 9(1), 8387 (2019)

10. Mastrandrea, R., Fournet, J., Barrat, A.: Contact patterns in a high school: a comparison between data collected using wearable sensors, contact diaries and friendship surveys. PLoS One 10(9), e0136497 (2015)

11. Michalski, R., Palus, S., Kazienko, P.: Matching organizational structure and social network extracted from email communication. In: International Conference on Business Information Systems, pp. 197-206. Springer (2011)

12. Newman, M.E.: Scientific collaboration networks. ii. Shortest paths, weighted networks, and centrality. Phys. Rev. E 64(1), 016132 (2001)

13. Pastor-Satorras, R., Castellano, C., Van Mieghem, P., Vespignani, A.: Epidemic processes in complex networks. Rev. Mod. Phys. 87(3), 925 (2015)

14. Peters, L.J., Cai, J.J., Wang, H.: Characterizing temporal bipartite networkssequential-versus cross-tasking. In: International Conference on Complex Networks and their Applications, pp. 28-39. Springer (2018)

15. Qu, C., Zhan, X., Wang, G., Wu, J., Zhang, Z.K.: Temporal information gathering process for node ranking in time-varying networks. Chaos: Interdisc. J. Nonlinear Sci. 29(3), 033116 (2019)

16. Rocha, L.E., Masuda, N.: Random walk centrality for temporal networks. New J. Phys. 16(6), 063023 (2014)

17. Stehlé, J., Voirin, N., Barrat, A., Cattuto, C., Isella, L., Pinton, J.F., Quaggiotto, M., Van den Broeck, W., Régis, C., Lina, B., et al.: High-resolution measurements of face-to-face contact patterns in a primary school. PLoS One 6(8), e23176 (2011)

18. Takaguchi, T., Sato, N., Yano, K., Masuda, N.: Importance of individual events in temporal networks. New J. Phys. 14(9), 093003 (2012)

19. Zhan, X.X., Hanjalic, A., Wang, H.: Information diffusion backbones in temporal networks. Sci. Rep. 9(1), 6798 (2019) 